OPEN ACCESS

Edited by:

Choong-Min Ryu,

Korea Research Institute

of Bioscience and Biotechnology,

South Korea

Reviewed by:

Brian H. Kvitko,

University of Georgia, United States

Fanhong Meng,

Texas A\&M University, United States

${ }^{*}$ Correspondence:

Wei Ding

dwing@swu.edu.cn

Specialty section: This article was submitted to Plant Microbe Interactions, a section of the journal

Frontiers in Microbiology

Received: 16 February 2017 Accepted: 19 June 2017

Published: 30 June 2017

Citation:

Yang L, Li S, Qin X, Jiang G, Chen J, Li B, Yao X, Liang $P$,

Zhang $Y$ and Ding W (2017)

Exposure to Umbelliferone Reduces

Ralstonia solanacearum Biofilm

Formation, Transcription of Type III

Secretion System Regulators

and Effectors and Virulence on

Tobacco. Front. Microbiol. 8:1234.

doi: 10.3389/fmicb.2017.01234

\section{Exposure to Umbelliferone Reduces Ralstonia solanacearum Biofilm Formation, Transcription of Type III Secretion System Regulators and Effectors and Virulence on Tobacco}

Liang Yang ${ }^{1}$, Shili Li1, Xiyun Qin², Gaofei Jiang 1,3, Juanni Chen', Bide Li1, Xiaoyuan Yao', Peibo Liang ${ }^{1}$, Yong Zhang ${ }^{4}$ and Wei Ding ${ }^{1 *}$

${ }^{1}$ Laboratory of Natural Products Pesticides, College of Plant Protection, Southwest University, Chongqing, China, ${ }^{2}$ Yunnan Academy of Tobacco Agricultural Research, Yuxi, China, ${ }^{3}$ Laboratoire des Interactions Plantes-Microorganismes, UMR441, Institut National de la Recherche Agronomique, Castanet-Tolosan, France, ${ }^{4}$ College of Resources and Environment, Southwest University, Chongqing, China

Ralstonia solanacearum is one of the most devastating phytopathogens and causes bacterial wilt, which leads to severe economic loss due to its worldwide distribution and broad host range. Certain plant-derived compounds (PDCs) can impair bacterial virulence by suppressing pathogenic factors of $R$. solanacearum. However, the inhibitory mechanisms of PDCs in bacterial virulence remain largely unknown. In this study, we screened a library of coumarins and derivatives, natural PDCs with fused benzene and $\alpha$-pyrone rings, for their effects on expression of the type III secretion system (T3SS) of $R$. solanacearum. Here, we show that umbelliferone (UM), a 7-hydroxycoumarin, suppressed T3SS regulator gene expression through HrpG-HrpB and PrhG-HrpB pathways. UM decreased gene expression of six type III effectors (RipX, RipD, RipP1, RipR, RipTAL, and RipW) of 10 representative effector genes but did not alter T2SS expression. In addition, biofilm formation of $R$. solanacearum was significantly reduced by UM, though swimming activity was not affected. We then observed that UM suppressed the wilting disease process by reducing colonization and proliferation in tobacco roots and stems. In summary, the findings reveal that UM may serve as a plant-derived inhibitor to manipulate $R$. solanacearum T3SS and biofilm formation, providing proof of concept that these key virulence factors are potential targets for the integrated control of bacterial wilt.

Keywords: R. solanacearum, type III secretion system, biofilm formation, coumarins, umbelliferone, inhibitor, bacterial wilt

\section{INTRODUCTION}

Ralstonia solanacearum, the causal agent of bacterial wilt, is one of the most devastating bacteria among the top 10 plant pathogens (Mansfield et al., 2012). R. solanacearum is a Gram-negative soil-borne bacterium that infects more than 250 plant species, invading its host from the soil through root openings and colonizing the root cortex. It rapidly reaches the xylem vessels where 
it grows to high cell densities, resulting in host wilting and death (González and Allen, 2003; Liu et al., 2005; Genin, 2010).

During the infection process, $R$. solanacearum utilizes many different virulence factors to cause disease in susceptible hosts, including the type III secretion system (T3SS), extracellular polysaccharides (EPS), extracellular proteins, motility activity, and biofilm formation (Saile et al., 1997; Tans-Kersten et al., 2001; Liu et al., 2005; Yao and Allen, 2007; Poueymiro and Genin, 2009). The main pathogenicity determinant in $R$. solanacearum is T3SS (Coll and Valls, 2013), which it deploys to secrete proteins directly inside the plant cell. These proteins, called type III effectors (T3Es), interact with molecules to manipulate plant cellular function, suppressing immunity and inducing the pathogen to multiply and spread (Macho, 2016; Mukaihara et al., 2016). Recently, many studies have demonstrated that expression of T3SS-associated genes is regulated by environmental factors, such as $\mathrm{pH}$, growth phase, temperature, nutrition, or cell density (Arlat et al., 1992; Wei et al., 1992; Van Dijk et al., 1999; Tang et al., 2006; Stauber et al., 2012). T3SS-associated genes are also regulated by natural-derived compounds or chemically synthesized compounds (Felise et al., 2008; Yang et al., 2008, 2014; Aiello et al., 2010; Duncan et al., 2012; Wu et al., 2015), making T3SS a particularly appealing target for the development of new agents for disease control. Because antimicrobial agents that utilize T3SSspecific inhibitors would affect pathogen virulence rather than viability, T3SS is also an attractive target for antimicrobial agents that generate low selective pressure for antimicrobial resistance development (Escaich, 2008; Rasko and Sperandio, 2010). Recently, certain plant phenolic compounds and their derivatives were found to inhibit T3SS in the plant pathogens Erwinia amylovora and Dickeya dadantii (Li et al., 2009, 2015; Khokhani et al., 2013). HrpB and ExsA, AraC class regulators, are important components of T3SS in bacteria that infect plants or animals. Recent study has identified T3SS inhibitors like $\mathrm{N}$-hydroxybenzimidazoles inhibits ExsA-dependent T3SS gene expression by interacting with the carboxy-terminal domain of ExsA (Marsden et al., 2016). As mentioned above, plant-derived compounds (PDCs) that inhibit T3SS expression in plant pathogens have recently been attracting increasing attention due to their potential abundant sources and environmental safety. Nonetheless, effective inhibitors need to be identified, and the regulation pathway of T3SS inhibitors remains largely uncharacterized.

Evidence to date suggests that $R$. solanacearum has evolved the ability to manipulate plant cell release or to degrade plant compounds, including galacturonic acid released by extracellular polygalacturonases from the plant cell walls, nourishing the bacteria during pathogenesis and with rapid disease onset (Allen et al., 1991; González and Allen, 2003). R. solanacearum is also proven to degrade plant salicylic acid (SA) to protect itself from inhibitory levels of this compound and also to enhance its virulence on the plant hosts like tobacco that use SA as a defense signal molecule (Lowe-Power et al., 2016). It has been observed that an exogenous compound could induce tomato resistance against $R$. solanacearum via overexpression of ethylene and jasmonic acid (Ghareeb et al., 2011). The
PDCs sclareol and cis-abienol are isolated from tobacco as inducers of $R$. solanacearum resistance (Seo et al., 2012). PDCs play an important role in inducing plant resistance to prevent $R$. solanacearum invasion, also play an important role in the interaction between the pathogen and host. However, the underlying mechanisms of PDCs such as coumarins on the virulence factors of plant pathogens remain unknown.

Coumarins, naturally derived compounds composed of fused benzene and $\alpha$-pyrone rings, have been shown to possess many biological activities, such as antibacterial, antifungal, anticoagulant, antioxidant, anticancer, and anti-inflammatory activity (Barot et al., 2015; Yang et al., 2016). Due to specific structural characteristics, some coumarins are regarded as phytoalexins biosynthesized by plant tissues in response to pathogenic infection that play a role in disease resistance (Andreae, 1948). Scopoletin is a phenolic coumarin and an important member of the group of phytoalexins isolated from many plants (Tal and Robeson, 1986). Umbelliferone (UM, 7-hydroxycoumarin) is a phytoalexin found in the roots of the sweet potato (Minamikawa et al., 1963) and Pharbitis nil (Yaoya et al., 2014). Plant-associated bacteria can metabolize UM (Parales and Harwood, 2002), and our previous studies indicated that hydroxycoumarins such as UM and daphnetin exert antibacterial activities against $R$. solanacearum and suppress expression of the T3SS-associated gene HrpG (Yang et al., 2016). As mentioned above, these results highlight the importance of investigating coumarins regulatory mechanisms in inhibition of T3SS expression in R. solanacearum.

Some PDCs can impair bacterial virulence by suppressing pathogenic factors of $R$. solanacearum. We hypothesized that coumarins, as specific PDCs, may alter expression of R. solanacearum pathogenic factor T3SS. In this study, a library of coumarins was screened for their effect on T3SS expression in $R$. solanacearum. We used quantitative realtime polymerase chain reaction (qRT-PCR) to investigate the molecular mechanism of UM, one of the best T3SS inhibitors, with regard to T3SS and T2SS gene expression in R. solanacearum. Furthermore, the effect of UM on the bacterial population in roots and stems and suppression of disease development in tobacco were investigated.

\section{MATERIALS AND METHODS}

\section{Materials and Bacterial Strains}

The bacterial wilt pathogen $R$. solanacearum CQPS-1 (phylotype I, race 1, biovar 3) and the RipX-lacZYA reporter strain CQPS-1 were used in this study. The reporter strain was constructed using the recombinant plasmid ppop3 as previously described (Zhang et al., 2011). R. solanacearum was incubated at $28^{\circ} \mathrm{C}$ in rich $\mathrm{B}$ medium or hydroponic plant culture medium supplemented with $2 \%$ sucrose [plant-sucrose (PS) medium; Yoshimochi et al., 2009].

The coumarins (HPLC $\geq 98 \%$ ) used in the study were purchased from Shanghai Yuanye Bio-Technology Co., Ltd. (Shanghai, China) and Adamas Reagent, Ltd. (Shanghai, China). 
Each compound was dissolved in dimethyl sulfoxide (DMSO) to a final concentration of $10 \mathrm{mg} / \mathrm{mL}$, and the compound solvent was added to the rich B or PS medium to prepare different concentrations of compound suspensions.

\section{$\beta$-Galactosidase Assay}

The effect of coumarins on expression of RipX was determined by measuring $\beta$-galactosidase activity of lac-ZYA reporter gene as previously described, with minor modifications (Zhang et al., 2011). The RipX-lacZYA reporter strain was inoculated in rich $\mathrm{B}$ medium for $6-7 \mathrm{~h}$ with shaking at $28^{\circ} \mathrm{C}$, and bacterial cells were collected. The bacterial suspension was transferred to PS medium supplemented with DMSO or $50 \mathrm{mg} / \mathrm{L}$ coumarins. When the $\mathrm{OD}_{600}$ of the bacterial suspension reached $0.1-0.2$, the $\beta$-galactosidase activity was measured. The enzyme assay was repeated two times.

\section{RNA Isolation and Quantitative Real-Time PCR}

Total RNA extraction and quantitative real-time PCR (qRT-PCR) were performed as previously described (Wu et al., 2015). For bacterial RNA analysis, an overnight-cultured $R$. solanacearum suspension was inoculated in PS medium supplemented with UM or DMSO and then incubated at $28^{\circ} \mathrm{C}$ with shaking at $180 \mathrm{rpm}$ for 6-7 h. The samples were centrifuged at $5000 \mathrm{rpm}$ for $10 \mathrm{~min}$ at $4^{\circ} \mathrm{C}$, and the supernatant was removed, the treated bacterial cells were collected. One microgram of cDNA was synthesized in a $20-\mu \mathrm{L}$ reaction mixture using the iScript cDNA synthesis kit (Bio-Rad, Hercules, CA, United States). The primers used for the tested genes were synthesized by BGI Technologies (Shenzhen, Guangzhou, China). All quantitative real-time PCR analyses were carried out in 96-well plates in a $20-\mu \mathrm{L}$ reaction system. Three technical replicate reactions were used for each sample. Normalized gene expression was calculated by Bio-Rad CFX, and SerC was used as a reference gene (Monteiro et al., 2012). All assays were performed three times in biological repeats.

\section{Biofilm Assay}

Biofilm formation of $R$. solanacearum supplemented with UM was performed in 96-well polystyrene microtiter plates as previously reported (Yao and Allen, 2007). Briefly, an overnight-cultured $R$. solanacearum suspension was inoculated in B medium supplemented with DMSO or different concentrations of UM ranging from 6.25 to $50 \mathrm{mg} \mathrm{L}^{-1}$. The samples were incubated at $30^{\circ} \mathrm{C}$ without shaking for 24 and $32 \mathrm{~h}$. Biofilms were stained with crystal violet, dissolved in 95\% ethanol and quantified by absorbance at $530 \mathrm{~nm}\left(\mathrm{OD}_{530}\right)$. All assays were carried out at least three times in biological repeats.

\section{Swimming Motility Assay}

The swimming motility of $R$. solanacearum was assessed on semi-solid motility media as previously reported (Kelman and Hruschka, 1973). Different concentrations of UM and DMSO were added to the semi-solid motility medium, and a $3-\mu \mathrm{L}$ overnight-cultured $R$. solanacearum suspension was dropped on the center of the plate. White halos were measured after $48 \mathrm{~h}$ cultivation at $30 \pm 1^{\circ} \mathrm{C}$, and motility was measured as the halo diameter. All assays were carried out in triplicate.

\section{Virulence Assay}

The naturalistic soil soak assay was used to evaluate the virulence of $R$. solanacearum after UM treatment. Briefly, unwound 6-week-old tobacco plants (Yunyan 87) were soaked in 25 or $50 \mathrm{mg} \mathrm{L}^{-1} \mathrm{UM}$ or DMSO. Individual plants were inoculated by pouring $20 \mathrm{~mL}$ of bacterial suspension into the soil to create a final inoculation density of $1 \times 10^{8} \mathrm{CFU} / \mathrm{g}$ soil. Inoculated plants were placed into a climate room at $28^{\circ} \mathrm{C}$ with a $14 \mathrm{~h} / 10 \mathrm{~h}$ light/dark cycle. Symptoms for each plant were scored daily, using a disease index scale from 0 to 4 (0 indicated no symptoms; 1 indicated $1-25 \%$ of leaves wilted; 2 indicated $26-50 \%$ of leaves wilted; 3 indicated $51-75 \%$ of leaves wilted; 4 indicated $76-100 \%$ of leaves wilted). Each treatment contained 16 plants in an independent experiment, and the inoculation assay was repeated three times.

The water-inoculation assay was used to evaluate the effect of UM treatment on the population of $R$. solanacearum in tobacco roots. We chose 4 -week-old tobacco plants, cleared the medium and placed the tobacco root into different concentrations of UM suspension for $5 \mathrm{~min}$. The plants were then placed into MS liquid medium supplemented with a $0.5 \% R$. solanacearum suspension. After four dips, the roots were collected, the root suspension was diluted from $10^{-1}$ to $10^{4}$, and $100 \mu \mathrm{L}$ was placed onto SMSA medium to quantify CFU.

To determine the bacterial population size in tobacco stems, $200 \mathrm{mg}$ of tissue at the base of the stems was destructively harvested. The tissue was ground in water and dilution plated onto SMSA medium for CFU quantification. Each treatment had two replications, and the entire experiment was performed three times. The semi-selective SMSA medium used in the assay was previously described (Elphinstone et al., 1996).

\section{RESULTS}

\section{The Effect of Coumarins on Expression of RipX in R. solanacearum}

Our primary research indicated that hydroxycoumarins such as UM and daphnetin have the potential to act as T3SS inhibitors because they significantly repress expression of HrpG (Yang et al., 2016). These results suggested that coumarins may inhibit expression of T3SS regulators and T3Es in R. solanacearum. To screen for coumarins that affect T3Es expression of in $R$. solanacearum, the RipX-lacZYA reporter strain was constructed for assessing $\beta$-galactosidase activity. Among the screened 17 plant-derived coumarins, (Supplementary Figure S1) six coumarins (coumarin, scoparone, UM, esculetin, daphnetin, and xanthotoxin) significantly inhibited RipX promoter activity (Figure 1). Compared with DMSO treatment, UM was the best inhibitor, with a 2.2-fold decrease in $\beta$-galactosidase activity. Therefore, UM was used for investigating mechanisms related to T3SS regulators and T3Es of R. solanacearum. 


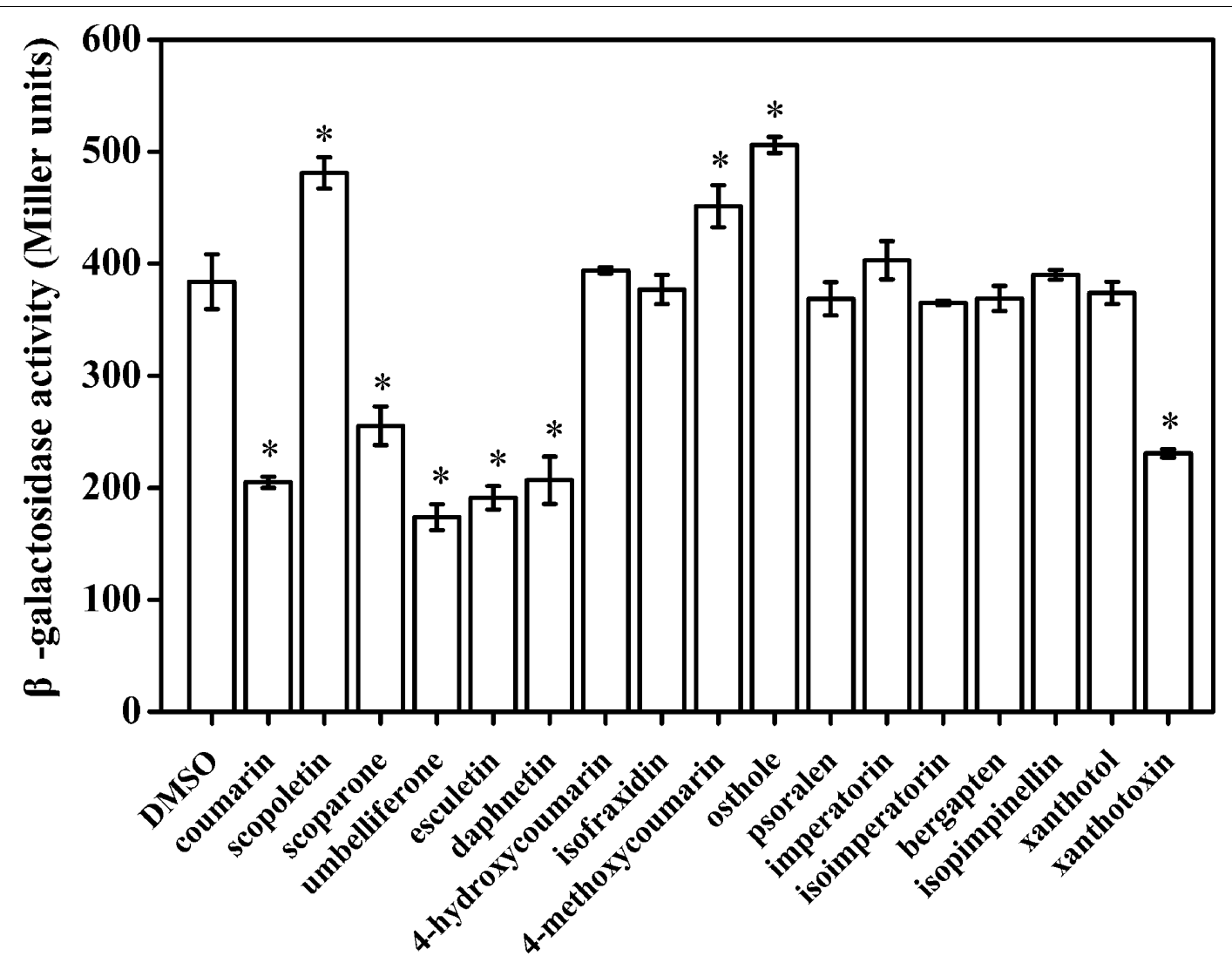

FIGURE 1 | The effect of coumarins on expression of RipX in $R$. solanacearum. Expression of $R$. solanacearum RipX was measured as $\beta$-galactosidase activity of the lacZYA reporter fusion gene in PS medium or PS medium supplemented with $50 \mathrm{mg} / \mathrm{L}$ of different coumarins. $R$. solanacearum was incubated in PS medium for 6-7 h. Once the $\mathrm{OD}_{600}$ of bacterial suspension was $0.1-0.2$, the promoter activities were determined. The coumarins were assayed two times, and error bars indicate the standard deviation. Asterisks indicate significant differences compared with DMSO treatment $(P<0.05$, Student's $t$-test).

\section{The Expression of RipX Is Inhibited by UM}

To further determine the inhibitory activity of UM on RipX expression, qRT-PCR was performed to investigate $R$. solanacearum expression at the transcriptional level in samples supplemented with different concentrations of UM, ranging from 6.25 to $50 \mathrm{mg} / \mathrm{L}$. Compared to the DMSO control, a significantly lower expression level of RipX mRNA was observed when the PS medium was supplemented with different concentrations of UM (Figure 2). At $50 \mathrm{mg} \mathrm{L}^{-1}$ of UM treatment, the transcription level was reduced by $80.67 \%$ compared to the DMSO control. The reduction in RipX expression in samples supplemented with $25,12.5$, and $6.25 \mathrm{mg} \mathrm{L}^{-1} \mathrm{UM}$ was 71.42 , 69.30 , and $56.06 \%$, respectively. These results demonstrate that the inhibitory activity of UM on transcriptional expression of RipX occurred in a concentration-dependent manner.

\section{UM Inhibits Expression of T3SS Regulators through the PrhG-HrpB and HrpG-HrpB Pathways}

$R i p X$ is a T3E gene, its expression is directly controlled by $\operatorname{HrpB}$, the expression of which is further regulated by $\operatorname{Hrp} G, \operatorname{Prh} G$ and other upstream regulators. As the suppression effect of UM on RipX expression was validated, we sought to determine how $\mathrm{UM}$ affects the regulatory components of other T3SS regulators, which have been well described in $R$. solanacearum (Peeters et al., 2013). To confirm this finding, mRNA levels of T3SS regulators were measured after DMSO or UM treatment. Our results showed that expression of some T3SS upstream regulators was significantly reduced, such as $\operatorname{Hrp} G, \operatorname{Prh} G$, and $\operatorname{HrpB}$, compared to the DMSO control (Figure 3). However, UM had no effect on expression of other T3SS upstream regulators (PrhA, PrhR, PrhI, and PrhJ). UM-mediated regulation of $R$. solanacearum T3SS regulators mainly occurred through the $\operatorname{HrpG}-\mathrm{HrpB}$ or $\operatorname{Prh} G-\operatorname{HrpB}$ pathway.

\section{UM Suppresses Expression of Most Tested Type III Effector Genes}

In $R$. solanacearum, $\operatorname{HrpB}$ is a downstream regulator in T3SS signal cascade and directly controls transcription of T3E genes. Based on initial experiments, we found that UM suppressed expression of both T3SS downstream regulatory gene $h r p B$ and the T3E gene RipX. To determine whether the suppression of $h r p B$ by UM results in transcriptional activation of other effector genes, qRT-PCR was performed to examine the mRNA levels of 


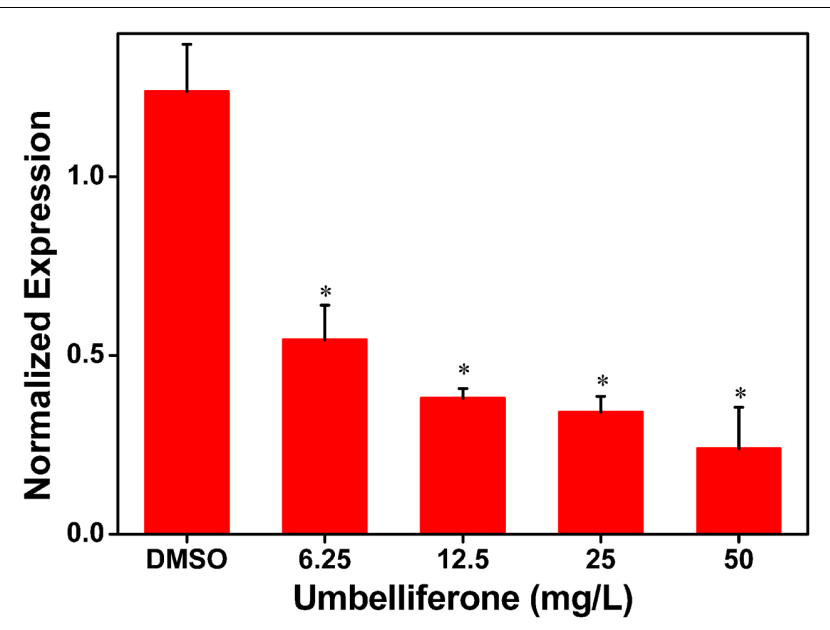

FIGURE 2 | UM inhibits expression of the type III representative effector RipX in $R$. solanacearum in a concentration-dependent manner. $R$. solanacearum cells incubated in PS medium were supplemented with different concentrations of UM, ranging from 6.25 to $50 \mathrm{mg} \mathrm{L}^{-1}$. After incubated 6-7 h, RipX expression of $R$. solanacearum was measures as the normalized expression using qRT-PCR. The $\Delta \Delta \mathrm{Cq}$ method was used to normalize the gene expression, and SerC was used as the reference gene. UM treatment inhibits expression of RipX two- to fourfold. Three biological replicates were performed, and error bars indicate the standard deviation. Asterisks indicate statistically differences between DMSO treatment and UM treatment $(P<0.05$, Student's $t$-test).

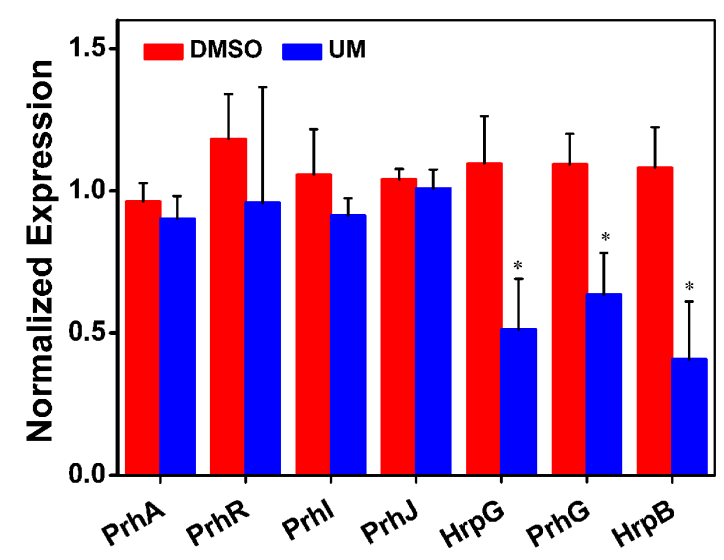

FIGURE 3 | The effect of UM on expression of T3SS pathway genes. Inhibition of $R$. solanacearum T3SS by UM occurs through the HrpG-HrpB and PrhG-HrpB pathways. Expression of T3SS pathway genes was evaluated by qRT-PCR in PS medium supplemented with DMSO or $50 \mathrm{mg} \mathrm{L}^{-1} \mathrm{UM}$. SerC was used as the reference gene to normalize gene expression using the $\triangle \triangle \mathrm{Cq}$ method. The results reflect three biological replicates, and error bars indicate the standard deviation. Asterisks indicate statistically differences in gene expression of bacterial cells supplemented with DMSO or $50 \mathrm{mg} \mathrm{L}^{-1}$ UM $(P<0.05$, Student's $t$-test $)$.

other effector genes in the presence and absence of UM. Because $R$. solanacearum has a large repertoire of effectors and it is rather difficult to evaluate the expression of all other effector genes, we chose 10 representative effector genes for this experiment. Based on the results, many of the tested T3Es genes were significantly

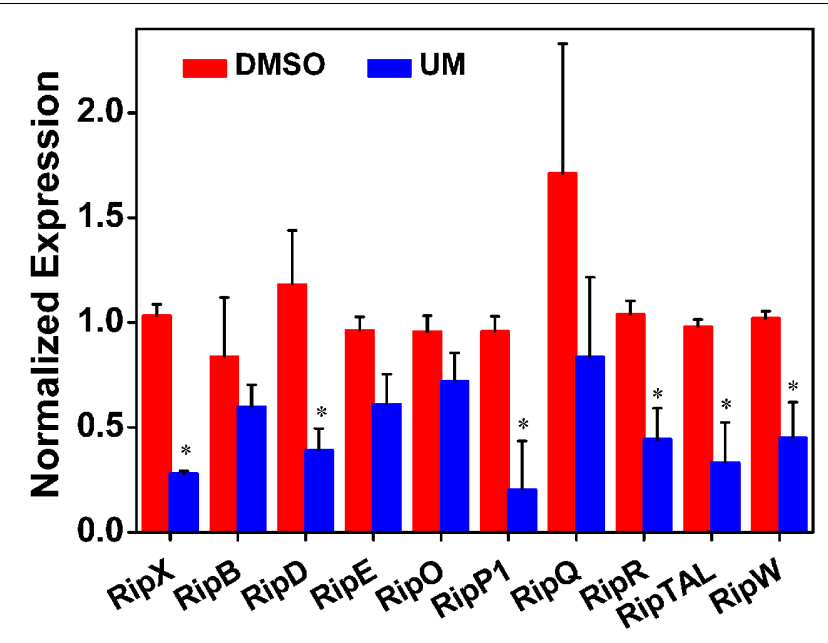

FIGURE 4 | Effect of UM on the inhibition of 10 representative type III effector genes in $R$. solanacearum cells. Expression of type III effector genes was measured by qRT-PCR in PS medium supplemented with DMSO or $50 \mathrm{mg} \mathrm{L}^{-1}$ UM. SerC was used as reference gene to normalize the type III effector genes expression using the $\Delta \triangle \mathrm{Cq}$ method. The results reflect three biological replicates and error bars indicate the standard deviation. Asterisks means the type III effector genes are significantly inhibited by UM treatment compared with DMSO treatment $(P<0.05$, Student's $t$-test).

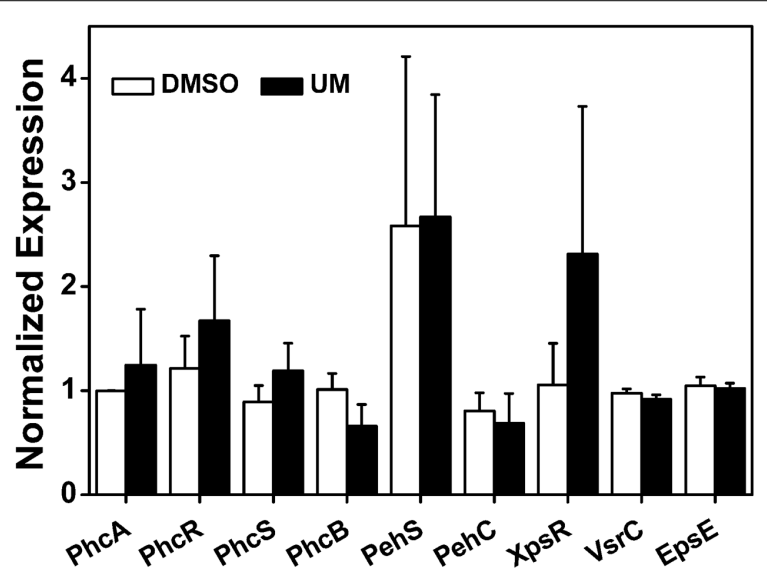

FIGURE 5 | Effect of UM on expression of other virulence-associated genes in $R$. solanacearum. qRT-PCR was performed to measure the relative expression level of virulence-associated genes in PS medium supplemented with DMSO or $50 \mathrm{mg} \mathrm{L}^{-1} \mathrm{UM}$. The assay was performed three biological replicates $(P<0.05$, Student's $t$-test).

suppressed by UM treatment, including RipX, RipD, RipP1, RipR, RipTAL, and RipW (Figure 4). Compared with DMSO treatment, the mRNA levels of T3Es genes, especially RipX, RipD, and RipP1, were significantly decreased three- to fourfold under UM treatment. In contrast, a few T3E genes, including RipB, $R i p E$, RipO, and RipQ, were similarly expressed in DMSO- or UM-treated cells. The results indicated that many other T3E genes may be suppressed by T3SS inhibitor UM.

To further evaluate the effect of UM on other virulence factors that play important roles at different infection stages, 
we measured the mRNA levels of the following: the quorum sensing system and the type II secretion system $P h c A$; the quorum sensing regulators $P h c B, P h c R, P h c S, P e h S$, and $P e h C$; the EPS secretion-related genes $X p s R$ and EPsE; and the swimming regulator gene $V s r C$. As shown in Figure 5, the nine tested virulence factor genes showed no significant differences between $\mathrm{UM}$ and DMSO treatments $(P<0.05)$. It appears that UM does not affect expression of most other virulence regulation genes.

\section{UM Reduces the Biofilm Formation of R. solanacearum}

To evaluate biofilm formation by $R$. solanacearum supplemented with UM, we used a standard polyvinyl chloride (PVC) microtiter plate assay using concentrations ranging from 6.25 to $50 \mathrm{mg} \mathrm{L}^{-1}$. As shown in Figure 6, UM treatment $50 \mathrm{mg} \mathrm{L}^{-1}$ significantly reduced biofilm formation by $R$. solanacearum $(\mathrm{P}<0.05)$. Compared with the control treatment, biofilm formation at $50 \mathrm{mg} \mathrm{L}^{-1}$ UM was also significantly reduced by 47.28 and $42.08 \%$ at 24 and $32 \mathrm{~h}$, respectively. The inhibitory activity of UM was concentration dependent. As the motility of $R$. solanacearum plays an important role in biofilm formation; the swimming motility of cells supplemented with UM was evaluated on semi-solid motility agar. After 2 days at $30^{\circ} \mathrm{C}$, motile colonies of $R$. solanacearum supplemented with UM at concentrations ranging from 6.25 to $50 \mathrm{mg} \mathrm{L}^{-1}$ were surrounded by a white halo with radiating streaks, producing swimming haloes similar to those the control treatment (Supplementary Figure S2), with no significant differences.

\section{UM Reduces the Virulence of Ralstonia solanacearum in Tobacco Plants}

Based on the strong suppressive activity of UM against biofilm formation and expression of T3SS regulators and T3Es genes in $R$. solanacearum, the effect of UM on bacterial wilt disease progression was evaluated. As shown in Figure 7C, compared with DMSO, UM at $50 \mathrm{mg} \mathrm{L}^{-1}$ altered the disease progress of bacterial wilt $(P<0.05)$. These findings suggest that $\mathrm{UM}$ reduces $R$. solanacearum virulence in tobacco plant by suppressing biofilm formation, expression of T3SS regulators and T3Es genes.

We further investigated the effects of UM on bacterial populations in tobacco roots and stems. As shown in Figure 7A, UM treatment significantly reduced $R$. solanacearum populations in tobacco roots in a concentration-dependent manner after incubated four dip in water inoculation assay. In addition, $50 \mathrm{mg} \mathrm{L}^{-1} \mathrm{UM}$ significantly reduced the population at the base of the tobacco plant stem. Compared with DMSO treatment, the pathogen populations of tobacco stems supplemented with $50 \mathrm{mg} \mathrm{L}^{-1} \mathrm{UM}$ were significantly reduced, by $21.18,21.96$, and $17.45 \%$ at 4,7 , and 10 days after inoculation, respectively (Figure 7B).

\section{DISCUSSION}

As a landmark discovery, advances in the study of bacterial virulence factors have provided evidence that T3SS is one of the main pathogenicity determinants in $R$. solanacearum

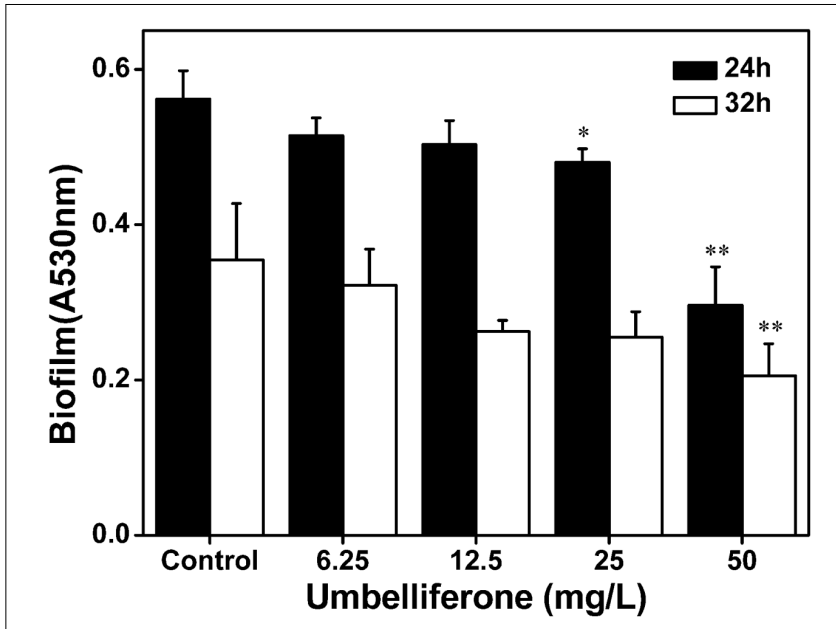

FIGURE 6 | Effect of UM on biofilm formation of $R$. solanacearum ranging from 6.25 to $50 \mathrm{mg} \mathrm{L}^{-1}$. Data shown are the means of independent experiments with a least three replicates. Bars represent standard errors of the means. Statistical significance was determined by Student's $t$-test in comparison with the DMSO control treatment $\left({ }^{*} P<0.05,{ }^{* *} P<0.01\right)$.

(Peeters et al., 2013). In the present study, 17 coumarins were evaluated for their ability to inhibit or induce expression of the R. solanacearum T3Es gene RipX (Figure 1). Our experiment revealed that six coumarins (coumarin, scoparone, UM, esculetin, daphnetin, and xanthotoxin) significantly inhibited expression of RipX and that three coumarins (scopoletin, 4-methoxycoumarin, and osthole) significantly induced RipX expression. We then chose a best T3SS inhibitor (UM) to investigate the mechanism of $R$. solanacearum T3SS regulation by PDCs. Further experiments demonstrated that UM suppresses expression of T3SS regulators through the PrhG-HrpB and $H r p G-H r p B$ pathways and inhibits expression of many T3Es genes (Figures 3, 4). Furthermore, UM suppressed biofilm formation without affecting swimming activity, and bacterial populations of $R$. solanacearum were reduced by UM treatment in the roots and stems of tobacco. In addition, UM reduced the virulence of $R$. solanacearum by suppressing biofilm formation as well as, expression of T3SS regulators and T3Es genes, resulting in delayed tobacco bacterial wilt disease progression (Figure 7).

Much evidence has suggested that screen compounds to target virulence factors is an effective strategy for controlling bacterial disease (Rasko and Sperandio, 2010; Wu et al., 2015). High-throughput screening is a powerful tool for identifying small molecule inhibitors that suppress expression of T3SS regulators (Pan et al., 2007). Recent studies have identified several classes of PDCs, as well as synthetic compounds as active T3SS inhibitors in a wide range of Gram-negative bacterial pathogens, including $R$. solanacearum, E. amylovora, D. dadantii, Xanthomonas oryzae, and Yersinia pestis (Jessen et al., 2014; Yang et al., 2014; Li et al., 2015; Lowe-Power et al., 2016). These PDCs determined to act as T3SS inhibitors include SA, p-coumaric acid, trans4-hydroxycinnamohydroxamic acid, 4-methoxycinnamic acid, benzoic acid, trans-2-phenylcyclopropane-1-carboxylic acid, 


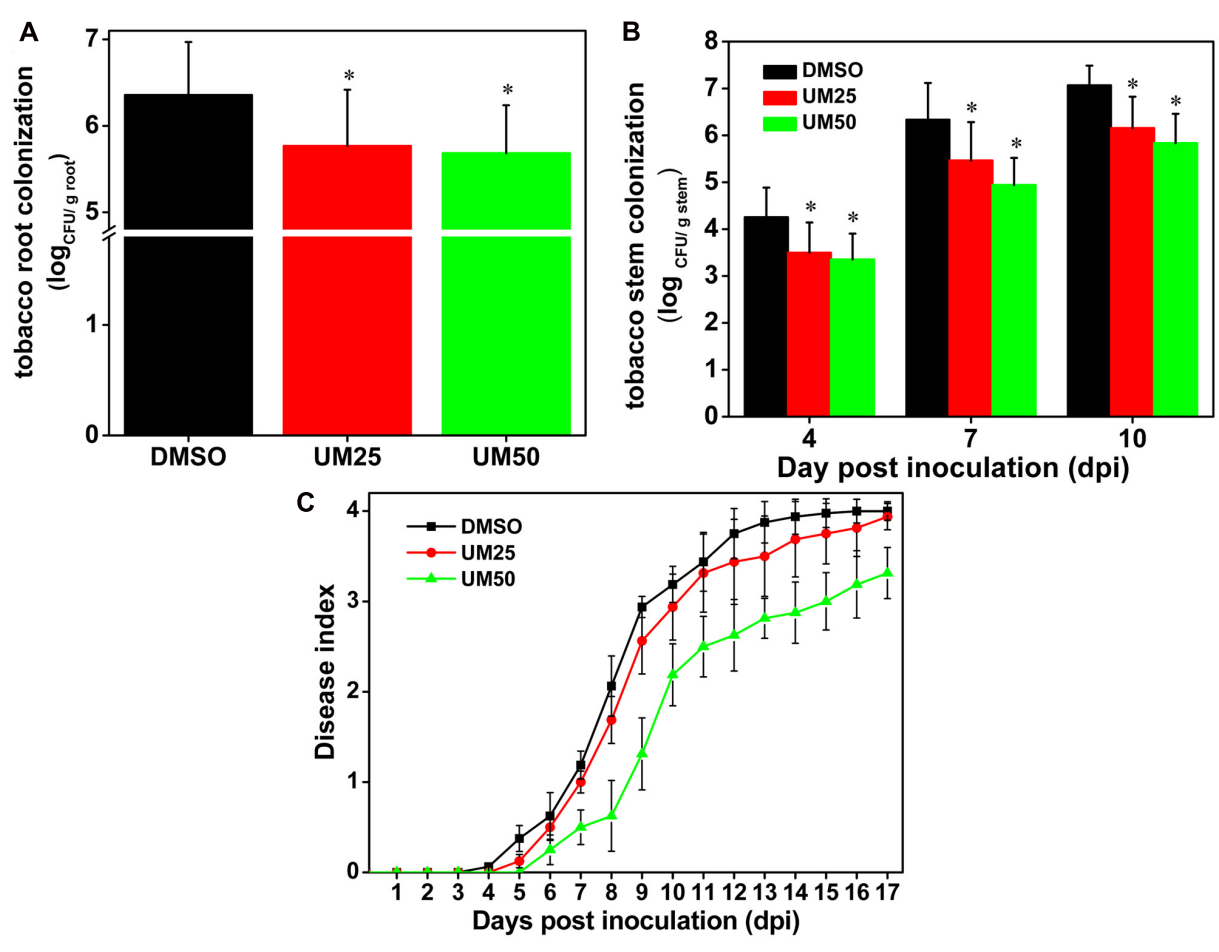

FIGURE 7 | UM treatment reduces virulence of $R$. solanacearum in tobacco. (A) Bacterial density in root tissue was quantified by dilution plating roots from water-inoculated plants. Asterisks indicate $P<0.05$ (Student's $t$-test). (B) $R$. solanacearum density in the stem was quantified by dilution tissue suspension from $10^{-1}$ to $10^{-4}$, with $100 \mu \mathrm{L}$ stem tissue suspension on SMSA medium. The plates were incubated in $30^{\circ} \mathrm{C}$ for 2 days, and the bacterial abundance was quantified in the plates. Each treatment included 10 plants, and the assay included three biological replicates. Asterisks indicate significant differences between the DMSO control and UM treatment $(P<0.05)$. (C) Effect of UM on the disease index of bacterial wilt in tobacco. Unwounded tobacco plants were soil-soak inoculated at a concentration of $20 \mathrm{~mL}\left(1 \times 10^{8} \mathrm{CFU} / \mathrm{mL}\right)$ and incubated at $30 \pm 1^{\circ} \mathrm{C}$ under a $14-\mathrm{h} / 10-\mathrm{h}$ light/dark cycle.

and $N$-(4-methoxycinnamyl)phthalimide (Li et al., 2009, 2015; Khokhani et al., 2013; Fan et al., 2017). In our study, six coumarins (coumarin, scoparone, UM, esculetin, daphnetin, and xanthotoxin) were found to significantly inhibit expression of the T3Es gene RipX (Figure 1), showing proof of concept that expression of T3SS in diverse bacterial pathogens can be inhibited by PDCs. Interestingly, T3SS expression in R. solanacearum and other bacteria can also be induced by certain PDCs, such as oleanolic acid, chlorogenic acid, scopoletin, taxifolin, $o$-coumaric acid, and trans-cinnamic acid (Yang et al., 2008; Wu et al., 2015). These studies suggest that PDCs may induce expression of T3SS in diverse bacterial pathogens, which is consistent with our finding that the $R$. solanacearum T3Es genes RipX was induced by some tested coumarins.

UM (7-hydroxycoumarin) is a coumarins compound distributed in a variety of plant species and has several promising biological activities, including anticoagulant, antioxidant, antibacterial, and antifungal (Barot et al., 2015). Our previous study proved that UM possesses antibacterial activity against $R$. solanacearum, with an $\mathrm{IC}_{50}$ of $96.88 \mathrm{mg} \mathrm{L}^{-1}$ when incubated for $24 \mathrm{~h}$ (Yang et al., 2016). It is interesting that UM was able to inhibit expression of T3SS regulator and T3Es genes in this study, expanding the biological functions of this special compound in plants. The T3SS regulator inhibitory activity of UM may be related to its specific structural characteristics. Previously, it has been demonstrated that plant phenolic compounds can serve as T3SS inhibitors or inducers (Khokhani et al., 2013), suggesting that different substitutions may be responsible for the same type of compounds exhibiting distinct T3SS activities. A strict requirement for the R-enantiomer at its stereocenter and tolerance for a variety of substituents on one of its two aromatic rings are key factors for the ability of a phenoxyacetamide series to function as T3SS inhibitors (Aiello et al., 2010). The para positioning of the hydroxyl group in the phenyl and the double bond of $p$-coumaric acid may be important for its T3SS inhibition activity (Li et al., 2009). Which is consistent with our finding that coumarins can act as T3SS inhibitors or inducers. Further analysis of several coumarins suggested that the hydroxyl-substituents of coumarins might be important for inhibiting expression of T3SS regulator and T3Es genes.

The T3SS regulator components and signaling cascade of R. solanacearum have been well characterized (Marenda et al., 1998; Aldon et al., 2000). PrhA, an outer membrane receptor at the top of the hrp regulatory pathway, is responsible for perceiving plant signals and activating downstream regulators. In this study, we found that UM inhibited expression of the downstream T3SS regulator HrpG, but it did not affect expression of upstream regulators genes, such as $\operatorname{Prh} A, \operatorname{Prh}$, PrhI, and PrhJ (Figure 3). Interestingly, HrpG activation of $R$. solanacearum cells was regulated only by the PrhA-PrhR-PrhI-PrhJ-HrpG 


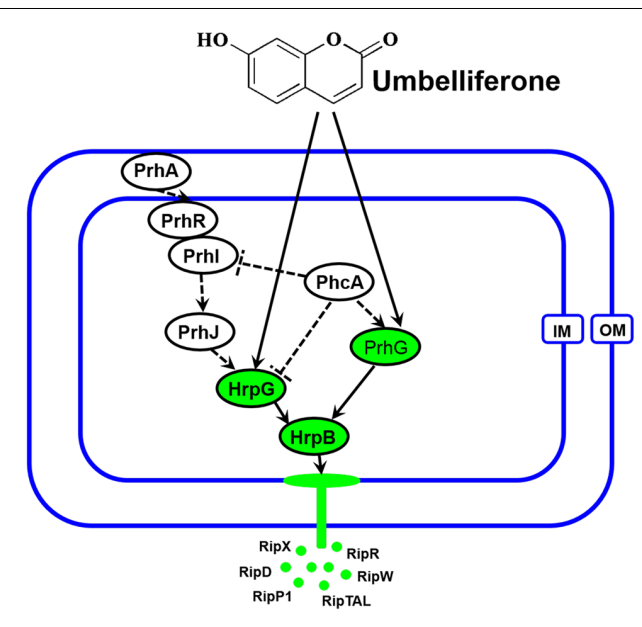

FIGURE 8 | Mode of UM in inhibiting $R$. solanacearum T3SS. The hrp gene expression in $R$. solanacearum is directly controlled by HrpB. HrpB is further controlled by two independent cascades involving PhcA-PrhG and PrhA-PrhR/Prhl-PrhJ-HrpG. The global regulator PhcA can also modulate T3SS expression through Prhl and HrpG (Peeters et al., 2013). In this study, we observed that T3SS inhibiter UM directly suppresses expression of HrpG and PrhG, inhibited the type III effector genes expression (e.g., RipX, RipD, RipW, RipR, RipTAL, and RipP1) through the HrpG-HrpB and PrhG-HrpB pathways. Ovals with green background indicate regulators inhibited by UM. Solid line arrows indicate direct induction of these regulators by UM. IM, inner membrane; OM, outer membrane.

pathway in a nutrient-rich medium. While, metabolic or plant signals affect the receptors on the outer membrane, activating the sensor kinase on the inner membrane and then activating the expression of $H r p G$ in nutrient-poor medium or plant co-cultivation experiments (Yoshimochi et al., 2009). Consistent with this previous study, our results suggested that expression of the T3SS regulator HrpG was significantly inhibited by UM and $R$. solanacearum may recognized UM as a metabolic signal to directly target T3SS downstream regulator HrpG. The hrp gene expression in $R$. solanacearum cells is also controlled by another T3SS regulator, PrhG, which occurs an independent pathway (Plener et al., 2010; Zhang et al., 2013). This regulator controls expression of T3SS under medium conditions but not in the presence of plant cells (Plener et al., 2010). Interestingly, we found that the T3SS regulator PrhG was also inhibited by UM in this study. Together, our results suggest that T3SS inhibitor UM inhibited expression of T3SS regulators and T3Es genes through the HrpG-HrpB and PrhG-HrpB pathways (Figure 8).

Recent studies have demonstrated that $R$. solanacearum utilizes T3SS to secrete T3Es to accelerate bacterial wilt progression. T3Es interact with molecules to manipulate plant cellular function, suppressing immunity and inducing pathogen multiplication and spread (Macho, 2016). In this study, we found that UM significantly suppressed the expression of some tested T3Es genes (RipX, RipD, RipP1, RipR, RipTAL, and RipW; Figure 4). Previously, it was demonstrated that half of effector genes are up-regulated in wilting tomato plants compared to expression in rich medium (Jacobs et al., 2012). This indicates that T3Es production is required during stages of bacterial wilt.
Similar to many plant pathogenic bacteria, $R$. solanacearum forms biofilms on plant roots, contributing to invasion and infection (Yao and Allen, 2007). Many studies have identified potential chemically synthesized or PDCs to target biofilm formation by pathogenic bacteria (O'Loughlin et al., 2013). In the current study, biofilm formation of $R$. solanacearum with $50 \mathrm{mg} \mathrm{L}^{-1} \mathrm{UM}$ was significantly reduced by 47.28 and $42.08 \%$ at 24 and 32 h, respectively (Figure 5). An ensuing inoculation assay using tobacco showed that UM treatment significantly reduced the $R$. solanacearum population in the roots and stems, and altered bacterial wilt disease progression (Figure 7). The findings suggest that UM reduces the virulence of $R$. solanacearum by suppressing biofilm formation and transcriptional expression of certain effector genes.

In summary, UM significantly reduced the biofilm formation by $R$. solanacearum without affecting swimming activity. The pathway by which UM regulates T3SS expression in $R$. solanacearum was first investigated. UM suppressed expression of T3SS regulators through the HrpG-HrpB and PrhG-HrpB pathways. In addition, T3Es genes RipX, RipD, and RipP1 were significantly decreased three- to fourfold under UM treatment. Finally, we observed that UM significantly reduces $R$. solanacearum population in the tobacco stem and suppresses the disease program of bacterial wilt. These results suggest that UM reduces the virulence of $R$. solanacearum by suppressing biofilm formation, transcription of the T3SS regulators and effectors. The findings indicated that UM has potential for use in the integrated control of plant bacterial wilt.

\section{AUTHOR CONTRIBUTIONS}

WD and LY conceived and designed the experiments. LY, XY, PL, and $Y Z$ performed the experiments. LY, WD, SL, and BL analyzed the data. WD, LY, XQ, JC, and GJ wrote and revised the paper.

\section{FUNDING}

The study was supported by the key project of the China National Tobacco Corporation (110201502019), Fundamental Research Funds for Central Universities (XDJK2017D166), and The National Natural Science Foundation of China (31572041).

\section{SUPPLEMENTARY MATERIAL}

The Supplementary Material for this article can be found online at: http://journal.frontiersin.org/article/10.3389/fmicb.2017. 01234/full\#supplementary-material

FIGURE S1 | The chemical structures of the tested coumarins.

FIGURE S2 | Effect of UM on the swimming motility of $R$. solanacearum. The halo diameter of typical bacterial halos formed on semi-solid agar plates. Experiments were repeated three times, with each replicate containing at least three plates for each concentration. Statistical significance was determined by Student's t-test in comparison with the DMSO control treatment. 


\section{REFERENCES}

Aiello, D., Williams, J. D., Majgier-Baranowska, H., Patel, I., Peet, N. P., Huang, J., et al. (2010). Discovery and characterization of inhibitors of Pseudomonas aeruginosa type III secretion. Antimicrob. Agents Chemother. 54, 1988-1999. doi: 10.1128/AAC.01598-09

Aldon, D., Brito, B., Boucher, C., and Genin, S. (2000). A bacterial sensor of plant cell contact controls the transcriptional induction of Ralstonia solanacearum pathogenicity genes. EMBO J. 19, 2304-2314. doi: 10.1093/emboj/19.10.2304

Allen, C., Huang, Y., and Sequeira, L. (1991). Cloning of genes affecting polygalacturonase production in Pseudomonas solanacearum. Mol. Plant Microbe Interact. 4, 147-154. doi: 10.1094/MPMI-4-147

Andreae, W. A. (1948). The isolation of a blue fluorescent compound scopoletin, from Green Mountain potato tubers, infected with leaf roll virus. Can. J. Res. 26, 31-34. doi: 10.1139/cjr48c-005

Arlat, M., Gough, C. L., Zischek, C., Barberis, P. A., Trigalet, A., and Boucher, C. A. (1992). Transcriptional organization and expression of the large hrp gene cluster of Pseudomonas solanacearum. Mol. Plant Microbe Interact. 5, 187-193. doi: 10.1094/MPMI-5-187

Barot, K. P., Jain, S. V., Kremer, L., Singh, S., and Ghate, M. D. (2015). Recent advances and therapeutic journey of coumarins: current status and perspectives. Med. Chem. Res. 24, 2771-2798. doi: 10.1007/s00044-015-1350-8

Coll, N. S., and Valls, M. (2013). Current knowledge on the Ralstonia solanacearum type III secretion system. Microb. Biotechnol. 6, 614-620. doi: 10.1111/17517915.12056

Duncan, M. C., Linington, R. G., and Auerbuch, V. (2012). Chemical inhibitors of the type three secretion system: disarming bacterial pathogens. Antimicrob. Agents Chemother. 56, 5433-5441. doi: 10.1128/AAC.00975-12

Elphinstone, J. G., Hennessy, J., Wilson, J. K., and Stead, D. E. (1996). Sensitivity of different methods for the detection of Ralstonia solanacearum in potato tuber extracts. EPPO Bull. 26, 663-678. doi: 10.1111/j.1365-2338.1996.tb01511.x

Escaich, S. (2008). Antivirulence as a new antibacterial approach for chemotherapy. Curr. Opin. Chem. Biol. 12, 400-408. doi: 10.1016/j.cbpa.2008.06.022

Fan, S., Tian, F., Li, J., Hutchins, W., Chen, H., Yang, F., et al. (2017). Identification of phenolic compounds that suppress the virulence of Xanthomonas oryzae on rice via the type III secretion system. Mol. Plant Pathol. 18, 555-568. doi: $10.1111 / \mathrm{mpp} .12415$

Felise, H. B., Nguyen, H. V., Pfuetzner, R. A., Barry, K. C., Jackson, S. R., Blanc, M. P., et al. (2008). An inhibitor of gram-negative bacterial virulence protein secretion. Cell Host Microbe 4, 325-336. doi: 10.1016/j.chom.2008.08.001

Genin, S. (2010). Molecular traits controlling host range and adaptation to plants in Ralstonia solanacearum. New Phytol. 187, 920-928. doi: 10.1111/j.1469-8137. 2010.03397.x

Ghareeb, H., Bozsó, Z., Ott, P. G., Repenning, C., Stahl, F., and Wydra, K. (2011). Transcriptome of silicon-induced resistance against Ralstonia solanacearum in the silicon non-accumulator tomato implicates priming effect. Physiol. Mol. Plant Pathol. 75, 83-89. doi: 10.1016/j.pmpp.2010.11.004

González, E. T., and Allen, C. (2003). Characterization of a Ralstonia solanacearum operon required for polygalacturonate degradation and uptake of galacturonic acid. Mol. Plant Microbe Interact. 16, 536-544. doi: 10.1094/MPMI.2003.16. 6.536

Jacobs, J. M., Babujee, L., Meng, F., Milling, A., and Allen, C. (2012). The in planta transcriptome of Ralstonia solanacearum: conserved physiological and virulence strategies during bacterial wilt of tomato. mBio 3:e00114-12. doi: $10.1128 / \mathrm{mBio} .00114-12$

Jessen, D. L., Bradley, D. S., and Nilles, M. L. (2014). A type III secretion system inhibitor targets YopD while revealing differential regulation of secretion in calcium-blind mutants of Yersinia pestis. Antimicrob. Agents Chemother. 58, 839-850. doi: 10.1128/Aac.01170-13

Kelman, A., and Hruschka, J. (1973). The role of motility and aerotaxis in the selective increase of avirulent bacteria in still broth cultures of Pseudomonas solanacearum. Microbiology 76, 177-188. doi: 10.1099/00221287-76-1-177

Khokhani, D., Zhang, C., Li, Y., Wang, Q., Zeng, Q., Yamazaki, A., et al. (2013). Discovery of plant phenolic compounds that act as type III secretion system inhibitors or inducers of the fire blight pathogen, Erwinia amylovora. Appl. Environ. Microbiol. 79, 5424-5436. doi: 10.1128/AEM.00845-13

Li, Y., Hutchins, W., Wu, X., Liang, C., Zhang, C., Yuan, X., et al. (2015). Derivative of plant phenolic compound inhibits the type III secretion system of Dickeya dadantii via HrpX/HrpY two-component signal transduction and Rsm systems. Mol. Plant Pathol. 16, 150-163. doi: 10.1111/mpp.12168

Li, Y., Peng, Q., Selimi, D., Wang, Q., Charkowski, A. O., Chen, X., et al. (2009). The plant phenolic compound p-coumaric acid represses gene expression in the Dickeya dadantii type III secretion system. Appl. Environ. Microbiol. 75, 1223-1228. doi: 10.1128/AEM.02015-08

Liu, H., Zhang, S., Schell, M. A., and Denny, T. P. (2005). Pyramiding unmarked deletions in Ralstonia solanacearum shows that secreted proteins in addition to plant cell-wall-degrading enzymes contribute to virulence. Mol. Plant Microbe Interact. 18, 1296-1305. doi: 10.1094/MPMI-18-1296

Lowe-Power, T. M., Jacobs, J. M., Ailloud, F., Fochs, B., Prior, P., and Allen, C. (2016). Degradation of the Plant defense signal salicylic acid protects Ralstonia solanacearum from toxicity and enhances virulence on tobacco. mBio 7:e0065616. doi: $10.1128 / \mathrm{mBio} .00656-16$

Macho, A. P. (2016). Subversion of plant cellular functions by bacterial typeIII effectors: beyond suppression of immunity. New Phytol. 210, 51-57. doi: 10.1111/nph.13605

Mansfield, J., Genin, S., Magori, S., Citovsky, V., Sriariyanum, M., Ronald, P., et al. (2012). Top 10 plant pathogenic bacteria in molecular plant pathology. Mol. Plant Pathol. 13, 614-629. doi: 10.1111/j.1364-3703.2012.00804.x

Marenda, M., Brito, B., Callard, D., Genin, S., Barberis, P., Boucher, C., et al. (1998). PrhA controls a novel regulatory pathway required for the specific induction of Ralstonia solanacearum hrp genes in the presence of plant cells. Mol. Microbiol. 27, 437-453. doi: 10.1046/j.1365-2958.1998.00692.x

Marsden, A. E., King, J. M., Spies, M. A., Kim, O. K., and Yahr, T. L. (2016). Inhibition of Pseudomonas aeruginosa ExsA DNA-binding activity by N-hydroxybenzimidazoles. Antimicrob. Agents Chemother. 60, 766-776. doi: 10.1128/AAC.02242-15

Minamikawa, T., Akazawa, T., and Uritani, I. (1963). Analytical study of umbelliferone and scopoletin synthesis in sweet potato roots infected by Ceratocystis fimbriata. Plant Physiol. 38, 493-497.

Monteiro, F., Genin, S., van Dijk, I., and Valls, M. (2012). A luminescent reporter evidences active expression of Ralstonia solanacearum type III secretion system genes throughout plant infection. Microbiology 158, 2107-2116. doi: 10.1099/ mic. $0.058610-0$

Mukaihara, T., Hatanaka, T., Nakano, M., and Oda, K. (2016). Ralstonia solanacearum type III effector RipAY is a glutathione-degrading enzyme that is activated by plant cytosolic thioredoxins and suppresses plant immunity. mBio 7:e00359-16. doi: 10.1128/mBio.00359-16

O’Loughlin, C. T., Miller, L. C., Siryaporn, A., Drescher, K., Semmelhack, M. F., and Bassler, B. L. (2013). A quorum-sensing inhibitor blocks Pseudomonas aeruginosa virulence and biofilm formation. Proc. Natl. Acad. Sci. U.S.A. 110, 17981-17986. doi: 10.1073/pnas.1316981110

Pan, N., Lee, C., and Goguen, J. (2007). High throughput screening for smallmolecule inhibitors of type III secretion in Yersinia pestis. Adv. Exp. Med. Biol. $603,367-375$.

Parales, R. E., and Harwood, C. S. (2002). Bacterial chemotaxis to pollutants and plant-derived aromatic molecules. Curr. Opin. Microbiol. 5, 266-273. doi: 10.1016/S1369-5274(02)00320-X

Peeters, N., Guidot, A., Vailleau, F., and Valls, M. (2013). Ralstonia solanacearum, a widespread bacterial plant pathogen in the post-genomic era. Mol. Plant Pathol. 14, 651-662. doi: 10.1111/mpp.12038

Plener, L., Manfredi, P., Valls, M., and Genin, S. (2010). PrhG, a transcriptional regulator responding to growth conditions, is involved in the control of the type III secretion system regulon in Ralstonia solanacearum. J. Bacteriol. 192, 1011-1019. doi: 10.1128/JB.01189-09

Poueymiro, M., and Genin, S. (2009). Secreted proteins from Ralstonia solanacearum: a hundred tricks to kill a plant. Curr. Opin. Microbiol. 12, 44-52. doi: 10.1016/j.mib.2008.11.008

Rasko, D. A., and Sperandio, V. (2010). Anti-virulence strategies to combat bacteria-mediated disease. Nat. Rev. Drug Discov. 9, 117-128. doi: 10.1038/ $\operatorname{nrd} 3013$

Saile, E., McGarvey, J. A., Schell, M. A., and Denny, T. P. (1997). Role of extracellular polysaccharide and endoglucanase in root invasion and colonization of tomato plants by Ralstonia solanacearum. Phytopathology 87, 1264-1271. doi: 10.1094/PHYTO.1997.87.12.1264

Seo, S., Gomi, K., Kaku, H., Abe, H., Seto, H., Nakatsu, S., et al. (2012). Identification of natural diterpenes that inhibit bacterial wilt disease in tobacco, 
tomato and Arabidopsis. Plant Cell Physiol. 53, 1432-1444. doi: 10.1093/pcp/ pcs085

Stauber, J. L., Loginicheva, E., and Schechter, L. M. (2012). Carbon source and cell density-dependent regulation of type III secretion system gene expression in Pseudomonas syringae pathovar tomato DC3000. Res. Microbiol. 163, 531-539. doi: 10.1016/j.resmic.2012.08.005

Tal, B., and Robeson, D. J. (1986). The metabolism of sunflower phytoalexins ayapin and scopoletin: plant-fungus interactions. Plant Physiol. 82, 167-172.

Tang, X., Xiao, Y., and Zhou, J. M. (2006). Regulation of the type III secretion system in phytopathogenic bacteria. Mol. Plant Microbe Interact. 19, 1159-1166. doi: $10.1094 / \mathrm{mpmi}$

Tans-Kersten, J., Huang, H., and Allen, C. (2001). Ralstonia solanacearum needs motility for invasive virulence on tomato. J. Bacteriol. 183, 3597-3605. doi: 10.1128/JB.183.12.3597-3605.2001

Van Dijk, K., Fouts, D. E., Rehm, A. H., Hill, A. R., Collmer, A., and Alfano, J. R. (1999). The Avr (effector) proteins HrmA (HopPsyA) and AvrPto are secreted in culture from Pseudomonas syringae pathovars via the Hrp (type III) protein secretion system in a temperature-and $\mathrm{pH}$-sensitive manner. J. Bacteriol. 181, 4790-4797.

Wei, Z. M., Sneath, B. J., and Beer, S. V. (1992). Expression of Erwinia amylovora hrp genes in response to environmental stimuli. J. Bacteriol. 174, 1875-1882. doi: 10.1128/jb.174.6.1875-1882.1992

Wu, D., Ding, W., Zhang, Y., Liu, X., and Yang, L. (2015). Oleanolic acid induces the type III secretion system of Ralstonia solanacearum. Front. Microbiol. 6:1466. doi: 10.3389/fmicb.2015.01466

Yang, F., Korban, S. S., Pusey, P. L., Elofsson, M., Sundin, G. W., and Zhao, Y. (2014). Small-molecule inhibitors suppress the expression of both type III secretion and amylovoran biosynthesis genes in Erwinia amylovora. Mol. Plant Pathol. 15, 44-57. doi: 10.1111/mpp.12064

Yang, L., Ding, W., Xu, Y., Wu, D., Li, S., Chen, J., et al. (2016). New insights into the antibacterial activity of hydroxycoumarins against Ralstonia solanacearum. Molecules 21:468. doi: 10.3390/molecules2104 0468
Yang, S., Peng, Q., San Francisco, M., Wang, Y., Zeng, Q., and Yang, C. H. (2008). Type III secretion system genes of Dickeya dadantii 3937 are induced by plant phenolic acids. PLoS ONE 3:e2973. doi: 10.1371/journal.pone.0002973.g001

Yao, J., and Allen, C. (2007). The plant pathogen Ralstonia solanacearum needs aerotaxis for normal biofilm formation and interactions with its tomato host. J. Bacteriol. 189, 6415-6424. doi: 10.1128/JB.00398-07

Yaoya, S., Kanho, H., Mikami, Y., Itani, T., Umehara, K., and Kuroyanagi, M. (2014). Umbelliferone released from hairy root cultures of Pharbitis nil treated with copper sulfate and its subsequent glucosylation. Biosci. Biotechnol. Biochem. 68, 1837-1841. doi: 10.1271/bbb.68.1837

Yoshimochi, T., Zhang, Y., Kiba, A., Hikichi, Y., and Ohnishi, K. (2009). Expression of $h r p G$ and activation of response regulator HrpG are controlled by distinct signal cascades in Ralstonia solanacearum. J. Gen. Plant Pathol. 75, 196-204. doi: 10.1007/s10327-009-0157-1

Zhang, Y., Chen, L., Yoshimochi, T., Kiba, A., Hikichi, Y., and Ohnishi, K. (2013). Functional analysis of Ralstonia solanacearum PrhG regulating the hrp regulon in host plants. Microbiology 159, 1695-1704. doi: 10.3389/fmicb.2015.00357

Zhang, Y., Kiba, A., Hikichi, Y., and Ohnishi, K. (2011). prhKLM genes of Ralstonia solanacearum encode novel activators of hrp regulon and are required for pathogenesis in tomato. FEMS Microbiol. Lett. 317, 75-82. doi: 10.1111/j.15746968.2011.02213.x

Conflict of Interest Statement: The authors declare that the research was conducted in the absence of any commercial or financial relationships that could be construed as a potential conflict of interest.

Copyright (c) 2017 Yang, Li, Qin, Jiang, Chen, Li, Yao, Liang, Zhang and Ding. This is an open-access article distributed under the terms of the Creative Commons Attribution License (CC BY). The use, distribution or reproduction in other forums is permitted, provided the original author(s) or licensor are credited and that the original publication in this journal is cited, in accordance with accepted academic practice. No use, distribution or reproduction is permitted which does not comply with these terms. 